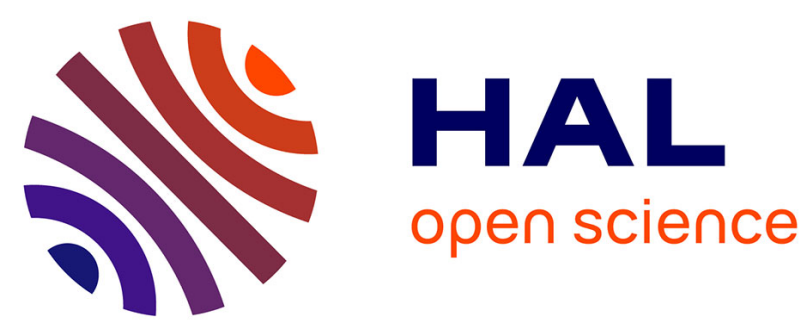

\title{
Diffusion in semi-dilute polymer solutions. A complementary experiment
}

\author{
T. Csiba, G. Jannink, Denys Durand, R. Papoular, A. Lapp, L. Auvray, \\ François Boué, J. Cotton, R. Borsali
}

\section{- To cite this version:}

T. Csiba, G. Jannink, Denys Durand, R. Papoular, A. Lapp, et al.. Diffusion in semi-dilute polymer solutions. A complementary experiment. Journal de Physique II, 1991, 1 (3), pp.381-396. 10.1051/jp2:1991175 . jpa-00247525

HAL Id: jpa-00247525

https://hal.science/jpa-00247525

Submitted on 1 Jan 1991

HAL is a multi-disciplinary open access archive for the deposit and dissemination of scientific research documents, whether they are published or not. The documents may come from teaching and research institutions in France or abroad, or from public or private research centers.
L'archive ouverte pluridisciplinaire HAL, est destinée au dépôt et à la diffusion de documents scientifiques de niveau recherche, publiés ou non, émanant des établissements d'enseignement et de recherche français ou étrangers, des laboratoires publics ou privés. 
Classification

Physics Abstracts

$6125 \mathrm{H}$

\title{
Diffusion in semi-dilute polymer solutions. A complementary experiment
}

\author{
T. Csiba, G. Jannink, D. Durand, R. Papoular, A Lapp, L Auvray, F. Boué, J. P. \\ Cotton and R. Borsali (*)
}

Laboratoire Léon Brillouin (CEA-CNRS), CEN-Saclay, 91191 Glf-sur-Yvette Cedex, France

(Recelved 5 September 1990, revised 4 December 1990, accepted 6 December 1990)

\begin{abstract}
Résumé. - La décroissance en temps des corrélations de concentration dans les solutions sem1dıluées de polydıméthylsıloxane a été mesurée à l'aide du spectromètre à écho de spin de neutrons au Laboratorre Léon Brillouın pour différentes valeurs du transfert de vecteur d'onde L'expénence a été effectuée successivement sur un échantillon homogène et sur un échantillon composé de deux parts égales de chaînes deutériées et non deutériées, à contraste moyen nul Nous donnons les relations de dispersion associées au mouvement brownien des chaînes, au volsınage de l'inverse de la distance de mallage, $1 / \xi$. Pour des valeurs de $q$ supérieures à $1 / \xi$, les résultats des deux expériences sont identiques, ma1s lorsque $q$ est inférieur à $1 / \xi$, Ils diffèrent On observe alors un mode coopératıf et un mode inter-diffusif Les valeurs des coefficients mesurés sont comparées aux théories de milieu effectıf et de solution diluée
\end{abstract}

\begin{abstract}
Relaxation times of concentration fluctuations in semi-dilute solutions of polydimethylsiloxane, have been measured at several values of the reciprocal wave vector $q$, with the neutron spin echo spectrometer of the Laboratoire Léon Brillouin The experiment has been carried out successively on a solute of identical chains, and on a solute divided in equal parts between labelled and non labelled chains at zero average contrast We report observations of the dispersion relation associated with the Brownian motion of the polymer chains, in the vicinity of the inverse mesh size $1 / \xi$ For values of $q$ which are greater than $1 / \xi$, the two experiments give identical results but when $q$ decreases below $1 / \xi$, the dispersion curves associated with each experiment are different $A$ bifurcation occurs at $1 / \xi$ and two distinct transport processes become observable, which are related to cooperative diffusion and to inter-diffusion respectively. The observed coefficients are compared with predictions of the effective medium and the dilute solution theories
\end{abstract}

\section{Introduction.}

Polymers in semi-dilute solution form a characteristic state [1] of condensed matter, which has been thorougly studied [2] during these last years. In particular, concentration diffusion has received great attention because it obeys a simple Fickian law, and because its coefficient has interestıng scaling properties. The dynamics of semi-dilute solutions is in fact a very rich field, in which characterıstic features of dilute solution and polymer melts are found [3]. On the

(*) CERMAV, Domaine Universitaire, Saint-Martın-d'Hères, 38400 Grenoble, France 
whole, three types of diffusion have been identified and characterized, namely cooperative diffusion, self-diffusion and inter-diffusion

Observation of these transport phenomena has been achieved in various ways analysis of the time dependence in the correlation function $[4,5]$ use of labelling techniques in forced Rayleigh scattering [6] and in neutron scattering [7]

Recently, Benoît et al. [8] improved the observation techniques, using an appropriate labelling strategy. Namely, dividing the solute in two fractions with different refractive index, and matching the solvent index so as to be intermediate between the two solute indices, a situation is obtained in which the average contrast is zero [9]. Under this condition the interdiffusion process can be isolated and detected in the scattering experiment.

This technique was applied with success to the determination of the inter-diffusion mode in ternary systems. Duval et al. [10] studied mixtures of two homopolymers in a solvent, using quasielastic light scattering. Benoît et al. [11] studied dilute solutions of diblock copolymers using neutron spin echo. As regards the experiment on the ternary polymer solution [10], the radiation source was light. the wave vector range is therefore limited to $q \leqslant q_{\max } \simeq 2 \times 10^{-3} \AA^{-1}$, and typically to $q \simeq 1 / R_{\mathrm{G}}$, where $R_{\mathrm{G}}$ is the radius of gyration of the polymer chain. An obvious extension of this investigation is to explore inter-diffusion at higher wave vector, 1.e in the range $q R_{\mathrm{G}}>1$, and especially around $q \xi=1$, where $\xi$ is the mesh size of the solute in the semi-dilute solution It will be of interest to determine what inter-diffusion exactly means at these higher values of $q$. Obviously, as $q$ increases beyond $1 / \xi$, all diffusion processes merge into the same process, associated with the motion of the chain internal modes. However, as $q$ decreases below $1 / \xi$, the chain overlap effects will become dominant and the observed dispersion relation between relaxation time and wave vector will depend upon the labelling structure

Experimental evidence for this process can partially be found in earlier observations [12] made with the neutron spin echo spectrometer, on samples containing a small fraction of labelled chains.

However, using the zero average contrast technique, the data which are presented here should give an improved insight into the problem.

\section{Description of the experiment.}

We describe here the polymer samples, the spectrometer and the formalism used to interpret the experiment.

2.1 The polymer solution is made of polydimethyls1loxane (PDMS) and toluene (T) The molecular masses and the concentration are given in table I. Two samples are examined. The first sample (no. 1) is a solution of (non deuterated) polydimethylsiloxane $\left(\mathrm{PDMS}_{\mathrm{H}}\right.$ ) chains of $N$ monomers, in deuterated toluene ( $\mathrm{T}_{\mathrm{S}}$ ) The second sample (no. 2) is a solution made of a mixture of fully deuterated polydimethylsiloxane chains $\left(\mathrm{PDMS}_{\mathrm{D}}\right.$ ) and non deuterated chains $\left(\mathrm{PDMS}_{\mathrm{H}}\right.$ ) (each of $N$ monomers) in a blend of deuterated and non deuterated toluene $\left(T_{D}, T_{H}\right)$. The number of (PDMS $)$ chains, $\mathbb{N} / 2$, is equal to the number of $\left(\right.$ PDMS $\left._{H}\right)$ chains, the number $\mathbb{N}$ being the total number of chains in experiment 1 , as well as in experiment 2 . The fraction $\alpha$ of deuterated toluene in sample 2, corresponds to the condition of zero average contrast of the solute. This is detailed in the next section.

2.2 SCATTERED INTENSITIES. - As a definition for the (coherent) scattered intensity $I(q, t)$ related to sample 1 , we use the following relation :

$$
I(q, t)=b^{2} C^{2} H(q, t)
$$


Table I. - Description of the samples and the polymer solutions Solvent. Toluene. Viscosity $\eta_{\mathrm{s}}=0.59 \times 10^{-2}$ poise, $\beta \eta_{\mathrm{s}}=1.46 \times 10^{11} \mathrm{~cm}^{-3} \mathrm{~s}$ Solute Polydimethylsiloxane (PDMS).

\begin{tabular}{|l|c|c|}
\hline & Non deuterated & Deuterated \\
\hline $\mathbf{M}_{\mathbf{w}}$ & 45000 & 44000 \\
$\mathrm{p}\left(=\mathrm{M}_{\mathrm{w}} / \mathrm{M}_{\mathrm{n}}\right)$ & 1.22 & 1.22 \\
$\mathrm{~N}$ & 610 & 550 \\
\hline
\end{tabular}

\section{Solutıon Polydimethylsıloxane in toluene}

\begin{tabular}{|l|l|l|}
\hline & Sample 1 (100\% sample) & Sample 2 (50\% sample) \\
\hline Mass concentration & $\rho_{\mathrm{H}}=0.2 \mathrm{~g} / \mathrm{cm}^{3}$ & $\begin{array}{l}\rho_{\mathrm{H}}=01 \mathrm{~g} / \mathrm{cm}^{3} \\
\rho_{\mathrm{D}}=0.1 \mathrm{~g} / \mathrm{cm}^{3}\end{array}$ \\
\hline Solvent & deuterated toluene & $\begin{array}{l}\text { mixture of deuterated and } \\
\text { non deuterated toluene } \\
(\alpha \text { (deuterated fraction) }=32 \%)\end{array}$ \\
\hline $\begin{array}{c}\text { Contrast of } \\
\text { monomer in solvent }\end{array}$ & $b=7.06 \times 10^{-12} \mathrm{~cm}$ & $\begin{array}{l}b_{\mathrm{Z}}=3.1 \times 10^{-12} \mathrm{~cm} \\
(\mathrm{zero} \text { average contrast condition) }\end{array}$ \\
\hline $\begin{array}{c}\text { Other important } \\
\text { characteristics }\end{array}$ & overlap concentration $\rho^{*}=0068 \mathrm{~g} / \mathrm{cm}^{3}$ \\
\hline
\end{tabular}

where $b$ is the contrast length between monomer and solvent molecules

$$
b=\mathfrak{B}_{\text {monomer }}-\mathfrak{B}_{\text {solvent }} \frac{\boldsymbol{v}_{\text {monomer }}}{\boldsymbol{v}_{\text {solvent }}}
$$

where $C$ is the concentration in monomers, $v_{\text {monomer }}$ the partial molar volume of the monomer and $v_{\text {solvent }}$ that of the solvent molecule

$$
C=\frac{\mathbb{N} N}{V}
$$

This is related to the mass concentration $\rho$ (Tab. I) $C=\rho \frac{\mathcal{A}}{m}$ (At, Avogadro number, $m$, molecular mass of the monomer).

The "intermediate » structure function $H(q, t)$ is defined by

$$
\begin{gathered}
H(q, t)=\frac{V}{(N \mathbb{N})^{2}} \sum_{a, b}^{N} \sum_{t \in a}^{N} \sum_{j \in b}^{N} f_{a l, b j}(q, t) \\
f_{a l, b j}(q, t)=\operatorname{Re}\left(\left\langle\exp \left\{i \mathbf{q} \cdot\left(\mathbf{r}_{a l}(0)-\mathbf{r}_{b j}(t)\right)\right\}\right\rangle\right)
\end{gathered}
$$


where $a, b$ are chain indices $(a, b=1, \ldots, \mathbb{N})$

where $t, j$ are monomer indices $(i, j=1, \ldots, N)$

where $\mathbf{r}_{a l}(0)$ is the position vector of monomer $t$ chain $a$, at time $t=0$.

The vector $\mathbf{q}$ is characterized by the scattering angle $\theta$ and has a modulus

$$
q=\frac{4 \pi}{\lambda} \sin \theta / 2
$$

which is the elastic momentum transfer ( $\lambda$ is the wavelength of the incident neutron radiation).

It will be useful to introduce the decomposition [2]

$$
H(q, t)=H^{\mathrm{I}}(q, t)+H^{\mathrm{II}}(q, t)
$$

where

$$
H^{\mathrm{I}}(q, t)=\frac{V}{(N \mathbb{N})^{2}} \sum_{a=b}^{N} \sum_{i, j}^{N} f_{a t, b j}(q, t)
$$

is the self contribution to the structure function (the dynamical form function of the chain) and where

$$
H^{\mathrm{II}}(q, t)=\frac{V}{(N \mathbb{N})^{2}} \sum_{a, b \neq}^{N} \sum_{i \in a}^{N} \sum_{j \in b}^{N} f_{a l, b j}(q, t)
$$

is the distinct contribution.

Note that [2]

$$
H(q, 0)=\int \mathrm{d}^{3} r \mathrm{e}^{l q \cdot r}\left\{\frac{\langle C(r) C(0)\rangle}{C^{2}}-1\right\}
$$

where $C(r)$ is the local concentration in monomers at point $r$.

For the case of sample 2, equation (1) writes

$$
\begin{aligned}
I(q, t)=\frac{C^{2}}{4}\left\{b_{\mathrm{D}}^{2} H_{\mathrm{DD}}(q, t)+b_{\mathrm{H}}^{2} H_{\mathrm{HH}}(q, t)+2\right. & \left.b_{\mathrm{D}} b_{\mathrm{H}} H_{\mathrm{DH}}(q, t)\right\} \\
& +\left(\mathfrak{B}_{\mathrm{TH}}-\mathfrak{B}_{\mathrm{TD}}\right)^{2} \alpha(1-\alpha) H_{\mathrm{T}}(q, t)
\end{aligned}
$$

Indices $\mathrm{D}, \mathrm{H}$ refer to the deuterated, non deuterated monomer; index $\mathrm{T}$ refers to the toluene molecule. The contrast lengths are given by the equation

$$
b_{\mathrm{D}, \mathrm{H}}=\mathfrak{B}_{\mathrm{D}, \mathrm{H}}-\left(\alpha \mathfrak{B}_{\mathrm{TD}}+(1-\alpha) \mathfrak{B}_{\mathrm{TH}}\right) \frac{v_{\mathrm{PDMS}}}{v} .
$$

The second term in (11) is the "Laue " contribution to the scattered intensity, caused by the presence of two solvents, with different indices. (This contribution is neglected).

Following equation (4) we write

$$
H_{\mathrm{DH}}=\frac{V}{(N \mathbb{N})^{2}} \sum_{a \in D}^{N} \sum_{b \in H}^{N} \sum_{i \in a}^{N} \sum_{j \in b}^{N} f_{a t, j b}(q, t)
$$

Zero average contrast implies $b_{\mathrm{D}}=-b_{\mathrm{H}}=b_{\mathrm{Z}}$ : this is obtaned when $\alpha=0.32$ in (12). Equation (11) now becomes

$$
I_{\mathrm{Z}}(q, t)=\frac{b_{\mathrm{Z}}^{2} C^{2}}{4}\left\{H_{\mathrm{DD}}(q, t)+H_{\mathrm{HH}}(q, t)-2 H_{\mathrm{DH}}(q, t)\right\}
$$


We introduce the decomposition (7), which writes here

$$
H_{\mathrm{DH}}=H_{\mathrm{D}}^{\mathrm{I}} \delta_{\mathrm{DH}}+H_{\mathrm{DH}}^{\mathrm{II}} \text {. }
$$

The self contribution will be expressed in the normalized form

$$
H_{\mathrm{D}}^{\mathrm{I}}(q, t)=\frac{N}{C} h(q, t)
$$

where $h(q, t)$ is the dynamical form function of the polymer chains. It obeys the condition

$$
h(0, t) \equiv 1 \text {. }
$$

We assume that this function is independent of labelling. Finally

$$
I_{\mathrm{Z}}(q, t)=b_{\mathrm{Z}}^{2} \frac{C^{2}}{4}\left\{2 \frac{N}{C} h(q, t)+\left(H_{\mathrm{DD}}^{\mathrm{II}}+H_{\mathrm{HH}}^{\mathrm{II}}-2 H_{\mathrm{DH}}^{\mathrm{II}}\right)\right\}
$$

If we assume that the interaction between $D$ and $H$ chains is identical to the interaction between $D$ and $D$ chains (and between $H$ and $H$ chains), then equation (17) simplifies and we obtain

$$
I_{\mathrm{Z}}(q, t)=b_{\mathrm{Z}}^{2} C N h(q, t) / 2 .
$$

Thus with zero average contrast, the scattered intensity is directly proportional to that scattered by a single chain, for static as well as for dynamic case.

Equation (18) will be used to interpret the data obtained with sample (2). The information is here complementary to the one derived in the first experiment. We note that $b_{\mathrm{Z}}$ in (18) and $b$ in (2) have the same magnitude (see Tab. I).

2.3 NEUTRON SPIN ECHO SPECTROMETER. - The scattered intensities relative to samples (1) and (2) are measured with the neutron spin echo spectrometer newly built in the Laboratoire Léon Brillouin. A detailed report of the experımental method is given in reference [13]. Polansed incident neutrons undergo Larmor precession while flying inside a first set of magnetic coils, interact with the sample, are reversed in spin before flying in a second set of colls. Interaction with sample produces a change of energy of the neutrons, that is a change of speed. It leads to a difference between the number of precessions before and after the sample as the field integrals $\int H \mathrm{~d} \ell$ are exactly opposite on the two sides. The resulting orientation $\delta \varphi$ of the spin is analysed on a mirror which gives an intensity $P_{\mathrm{f}}=P_{1} \cos \delta \varphi$

For a given value of the wavelength $\lambda$ of the incident neutron, sum over all energy transfers $\omega$ gives :

$$
P_{\mathrm{f}}=P_{1} \int S(q, \omega) \cos \delta \varphi \mathrm{d} \omega=P_{1} \int S(q, \omega) \cos (\omega t(\lambda)) \mathrm{d} \omega
$$

a Fourier transform in time which can be written

$$
P_{\mathrm{f}}=\frac{I(q, t)}{I(q, 0)}
$$

where the «Fourier time » is

$$
t(\lambda)\{s\}=1.8635 \times 10^{-14} \lambda^{3}\{\AA\} \int H \mathrm{~d} \ell\{\text { Oe.m }\} .
$$


The spin echo allows the use of a large distribution of wavelengths. The mean value is here $\lambda=654 \AA$; with a scattering angle interval :

$$
2^{\circ}<\theta<5^{\circ}
$$

this gives

$$
3.3 \times 10^{-2} \AA^{-1}<q<8.5 \times 10^{-2} \AA^{-1} .
$$

Here the half width of the wavelength distribution is $\Delta \lambda / \lambda \sim 8 \%$ and the angular collimation by two holes of $25 \mathrm{~mm}$ at $6 \mathrm{~m}$ give the $q$ resolution:

$$
\frac{\Delta q}{q}=\left[\left(\frac{\Delta \lambda}{\lambda}\right)^{2}+\left(\frac{\Delta \theta}{\theta}\right)^{2}\right]^{1 / 2}=\left(0.0064+\frac{0.035}{\theta^{2}}\right)^{1 / 2} .
$$

The intensity scattered by the solvent alone is found to be negligible for both samples.

\section{Expected behaviours for semi-dilute solutions.}

The time dependence of the dynamical structure functions $H(q, t)$ (Eq (7)), is given by the Brownian motion of the polymer chains. The diffusion coefficient related to a dynamical process described by a function $H(q, t)$, can always be defined formally by considering the first cumulant approximation [16] :

$$
H(q, t)=H(q, 0) \exp \left\{-{ }^{\circ} \Gamma_{q} t\right\}
$$

where ${ }^{\circ} \Gamma_{q}$ is the inverse of the relaxation time. We can write

$$
{ }^{\circ} \Gamma_{q}={ }^{\circ} D q^{2}
$$

which defines an apparent diffusion coefficient. This coefficient can be $q$ dependent. In polymer solutions, there are several diffusion processes, and to each of them a diffusion coefficient is ascribed A detalled presentation is found in references [16] and [19]. Here we give a summary of the important diffusion types of transport in sem1-dilute solutions

3.1 Diffusion OF A MASS POINT. - Self diffusion of a mass point is defined by the velocity $v(t)$ autocorrelation function

$$
D_{\text {self }}=\frac{1}{3} \int_{0}^{\infty} \mathrm{d} t\langle\mathbf{v}(0) \cdot \mathbf{v}(t)\rangle
$$

This definition applies in particular to the center of mass of a polymer chain. For sem1-dilute solutions the expression for the center of mass self diffusion is [19]

$$
D_{\text {self }}=\frac{1}{6 \pi \eta_{s} \beta R_{\mathrm{H}}\left(C / C^{*}\right)^{(2-\nu) /(3 \nu-1)}}
$$

where $\eta_{\mathrm{s}}$ is the solvent viscosity, $R_{\mathrm{H}}$ the hydrodynamic radius, $\nu$ the swelling exponent and $C^{*}$ the overlap concentration $\left(C^{*}=\frac{N}{2^{3 / 2} R_{\mathrm{G}}^{3}}\right.$, where $R_{\mathrm{G}}$ is the radius of gyration at zero concentration). This coefficient is determined by scattering experiments in the range $q R_{\mathrm{H}}<1$

The case of a mass point belonging to a flexible polymer chain requires a more detailed analysis. Here namely the mass point belongs to a fractal structure and this structure diffuses 
in Euclidean space. A geometrical transformation is required and this implies a formulation of the diffusion constant in combination of elementary units which is different from the conventional one. In the free draining limit ("Rouse » regime), one defines the coefficient [16]

$$
\mathcal{D}=\frac{R_{\mathrm{G}}^{2}}{\beta \zeta N} \quad \text { in units }\left(\mathrm{cm}^{4} / \mathrm{s}\right)
$$

where $\zeta$ is the friction coefficient of a monomer. In the case where the solvent hydrodynamic interaction is important ( Zimm » regime), one defines a coefficient [18]

$$
\mathfrak{D}=\frac{1}{6 \pi \eta_{\mathrm{s}} \beta} \quad \text { in units } .\left(\mathrm{cm}^{3} / \mathrm{s}\right)
$$

These coefficients appear naturally in the incoherent cross section. They are also found in the coherent cross section which we consider here.

3.2 RELAXATION OF THE POLYMER FORM FUNCTION. - Here we consider asymptotic behaviours and first cumulant approximations.

In the range $q \xi \leqslant 1$, excluded volume and hydrodynamic interactions are screened. We expect, for sample (2) and $q R_{\mathrm{G}}>1$

$$
h(q, t)=h(q, 0) \exp \left\{-\frac{2}{\sqrt{\pi}}\left({ }^{\mathrm{R}} \Gamma_{q} t\right)^{1 / 2}\right\}
$$

where

$$
{ }^{\mathrm{R}} \Gamma_{q}=\mathfrak{D} q^{4} .
$$

In the first cumulant approximation (21), ${ }^{\circ} \Gamma_{q}={ }^{\mathrm{R}} \Gamma_{q}$.

In the range $q \xi \geqslant 1$, the observed mass points behave as if they belonged to an isolated chain in the dilute regime The hydrodynamic interaction is here fully developed. We expect for sample (2)

$$
h(q, t)=h(q, 0) \exp \left\{-135\left({ }^{\mathrm{z}} \Gamma_{q} t\right)^{2 / 3}\right\}
$$

where

$$
\mathrm{z}_{q}=\mathfrak{D} q^{3}
$$

In the first cumulant approxumation (21), ${ }^{\circ} \Gamma_{q}={ }^{\mathrm{z}} \Gamma_{q}$.

Equation (27b) also modelizes the dynamics of sample (1) for $q \xi \geqslant 1$, and we write

$$
C H(q, t)=N h(q, t)
$$

The other diffusion processes involve more than one chain and are therefore cooperative.

33 COOPERATIVE DIFFUSION SOLUTE AGAINST SOLVENT - The concentration diffusion of the total monomer concentration is characterized by a diffusion coefficient $[18,19]$

$$
D_{\text {coop }}=\frac{1}{\zeta} \frac{\partial \pi}{\partial C}=\frac{1}{6 \pi \eta_{\mathrm{s}} \xi_{\mathrm{H}} \beta} \quad \text { in units }\left(\mathrm{cm}^{2} / \mathrm{s}\right)
$$

where $\zeta$ is the friction coefficient and $\pi$ the osmotic pressure, $\xi_{H}$ being the hydrodynamic mesh size. 
With sample (1), and for $q \xi_{H} \leqslant 1$, we expect to observe

$$
H(q, t)=H(q, 0) \exp \left(-\Gamma_{q} t\right)
$$

with

$$
\Gamma_{q}=D_{\text {coop }} q^{2}
$$

as for a «Fickian » process.

3.4 INTERDIFFUSION. - This is the object of our complementary experiment (sample (2)). The quantity given by (17) is actually the Fourier transform of

$$
\left\langle\left[C_{\mathrm{H}}(r=0, t=0)-C_{\mathrm{D}}(r=0, t=0)\right]\left[C_{\mathrm{H}}(r, t)-C_{\mathrm{D}}(r, t)\right]\right\rangle
$$

where $C_{\mathrm{H}}(r, t)$ and $C_{\mathrm{D}}(r, t)$ are the local concentration of monomers of species $\mathrm{H}$ and $\mathrm{D}$ respectively at time $t$ Interdiffusion is exactly described by this expression [20] In the limit $q \rightarrow 0$, it is characterized by a diffusion coefficient, which gives the flux associated with the concentration gradient of a given solute fraction It will be of interest to observe what the interdiffusion process means in the vicinity $q \simeq 1 / \xi$.

Since however we consider here that both polymer species are physically identical, it is easy to see that interdiffusion amounts to self-diffusion

\section{Results.}

We first report the results of small angle scattering experiments aimed at the determination of the static structure function of samples (1) and (2). These results are of interest by themselves; but they also give us an experımental proof that sample (2) satisfies the zero average contrast condition (14).

Subsequently, we give the time dependences of the dynamical structure functions observed on samples (1) and (2), which is the main purpose of this paper.

4.1 STATIC STRUCTURE FUNCTION. - Scattered intensities were measured at the spectrometer PACE of the Laboratoire Léon Brillouın. In figure 1, after substraction of the background, intensities $I(q, 0)$ (Eq. (1)) and $I_{Z}(q, 0)$ (Eq. (18)) are plotted against $q$. These two curves correspond respectively to sample (1) and (2).

The form of $I_{Z}(q, 0)$ matches a "Debye " function, with one unknown parameter, $R_{\mathrm{G}}$, the radius of gyration. (This function reads $\frac{2}{x^{2}}\left(\mathrm{e}^{-x}-1+x\right), x=q^{2} R_{\mathrm{G}}^{2}$.) We find $R_{\mathrm{G}}=70 \pm 3 \AA$.

The form of the function $I(q, 0)$ is Lorentzian, with one unknown parameter, the screening length $\xi$. (The function reads $\left.\left(q^{2}+\xi^{-2}\right)^{-1}\right)$ The best fit gives $\xi=10 \pm 1 \AA$.

The ratio $I(q, 0) / I_{\mathrm{Z}}(q, 0)$ satisfies a sum rule, which writes [2]

$$
\frac{b_{\mathrm{Z}}^{2} I(q \rightarrow 0,0)}{b^{2} I_{Z}(q \rightarrow 0,0)}=\left(\frac{C}{N}\right) \frac{3 \nu-1}{3 \nu} \frac{1}{F_{\infty}}\left(\frac{\xi}{\Gamma}\right)^{3}
$$

where $\nu, F_{\infty}, \Gamma$ are characteristic universal coefficients and where $b, b_{\mathrm{Z}}$ are given in table 1. The theoretical value of the above ratio is 0092 In order to test equation (31) we have to normalize $I$ and $I_{\mathrm{Z}}$ with respect to each other. This was achieved by superimposing the tails $\left(q>0.1 \AA^{-1}\right)$. The value of the ratio (31) obtained in this manner is $0.085 \pm 0.02$. Thus the results displayed in figure 1 prove that sample (2) satisfies zero average contrast. 


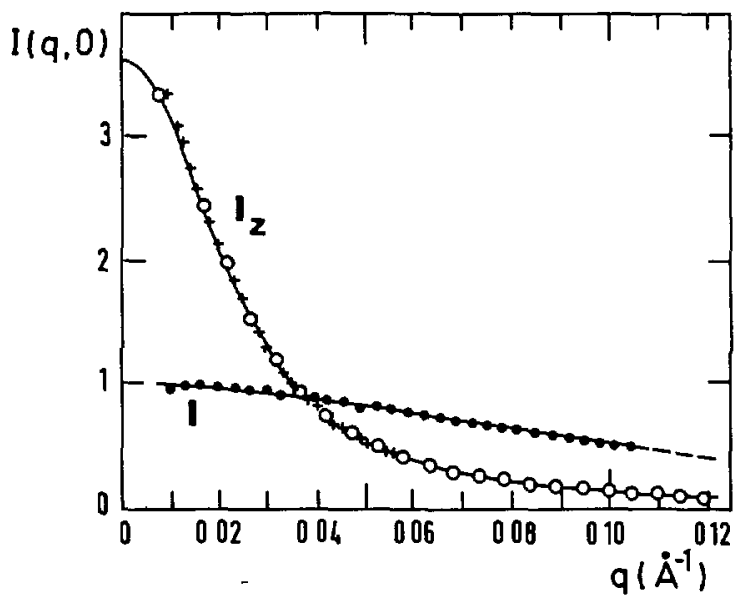

Fig 1. - Results of the small angle scattering experiment Intensities $I_{Z}(q, 0)$ corresponding to measurements obtained in two different $q$ ranges (symbols $O$ and,$+\left(I_{z}\right)$ ) and $I(q, 0)$ (symbol $\bullet,(I)$ ) are plotted against $q$ in arbitrary units The full line is the Debye function which fits the data for the zero mean contrast solution (sample 2) while the dashed line is the Lorentzian form which fits the data of sample 1. The solution is semi-dilute both in cases 1 and 2 . The quantities denived from these fits are respectively the radius of gyration $R_{\mathrm{G}}$ of one chain and the screenıng length $\xi$ at the same concentration $\rho=02 \mathrm{~g} \mathrm{~cm}^{-3}$

4.2 DYNAMIC STRUCTURE FUNCTIONS TIME DEPENDENCE OF THE CORRELATION FUNCTIONS. - The correlation functions tend to zero as $t \rightarrow \infty$ : the nature of the decay reveals the dynamics of the polymer chains. Depending on the diffusion process, the decay may be represented by a single exponential, a sum of exponentials or a stretched exponential

We represent the results $I(q, t) / I(q, 0)$ as a function of $t$ in different ways, in order to point out the specific behaviours which are observed. Differences arise in relation to the nature of the samples 1$)$ and 2$)$, and the wave vector range $(q \xi \leqslant 1$ or $\geqslant 1)$.

Figures 2a, 2b display:

a) the relaxation function $I(q, t) / I(q, 0)=H(q, t) / H(q, 0)$ associated with the total monomer concentration diffusion in the range $q \xi<1$ (sample (1), Eq. (1)). In these conditions the relaxation corresponds to a Fickian diffusion process [19]. We therefore expect this function to be exponential and in this case, $\log I(q, t)$ is a linear function of $t$ (see Eq. (21)) ;

b) the relaxation function observed on sample (2), at the same values of $q$ is not of the exponential type It is expected [19] to that the dynamics of the single chain in the solution is described by a "Rouse »-like motion in an effective medium (see Eq. (27a)) Therefore, the data are compared to a stretched exponential decay, with exponent $1 / 2$.

Figure $2 \mathrm{c}$ displays the relaxation functions which are observed on samples (1) and (2), for $q=0.074 \AA^{-1}$ (in the range $q \xi>1$ ). The observation is now made at a scale within the mesh size $\xi$. We expect to observe, in both samples, the diffusion of the polymer internal modes . here $H^{\mathrm{II}}(q, t)$ does not contribute and as a consequence

$$
I(q, t) / I(q, 0)=h(q, t) .
$$

Equation (27b) predicts a stretched exponential decay of $h(q, t)$ with an exponent $2 / 3$ for 


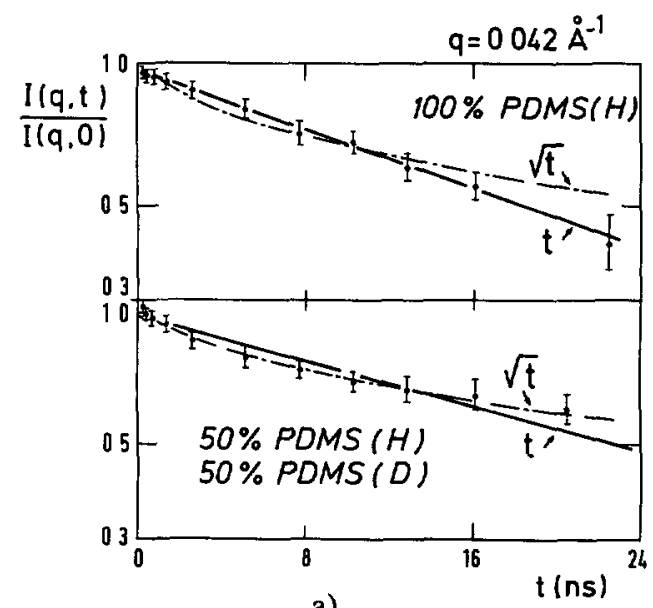

a)

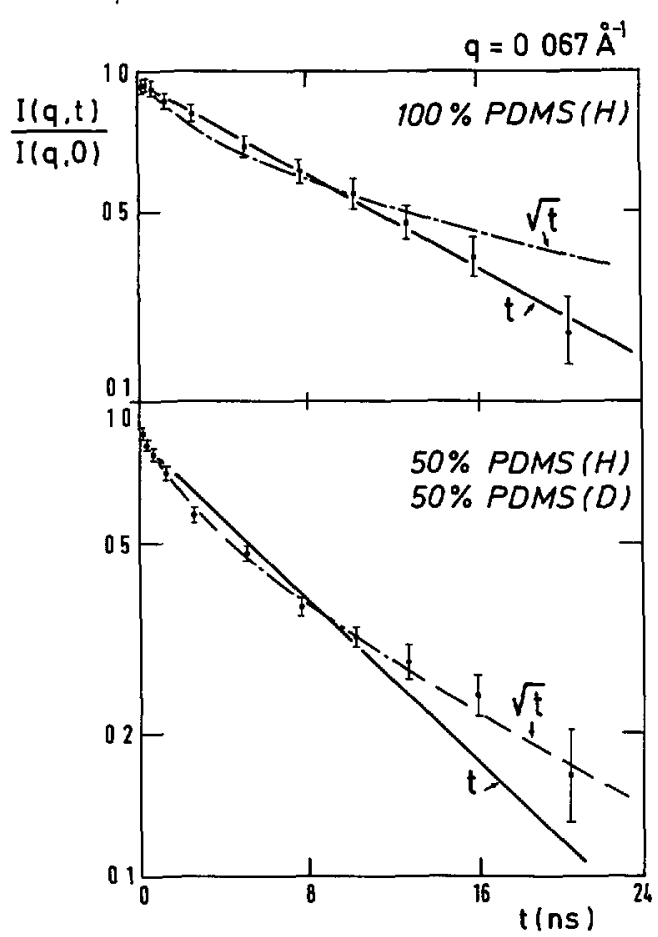

b)

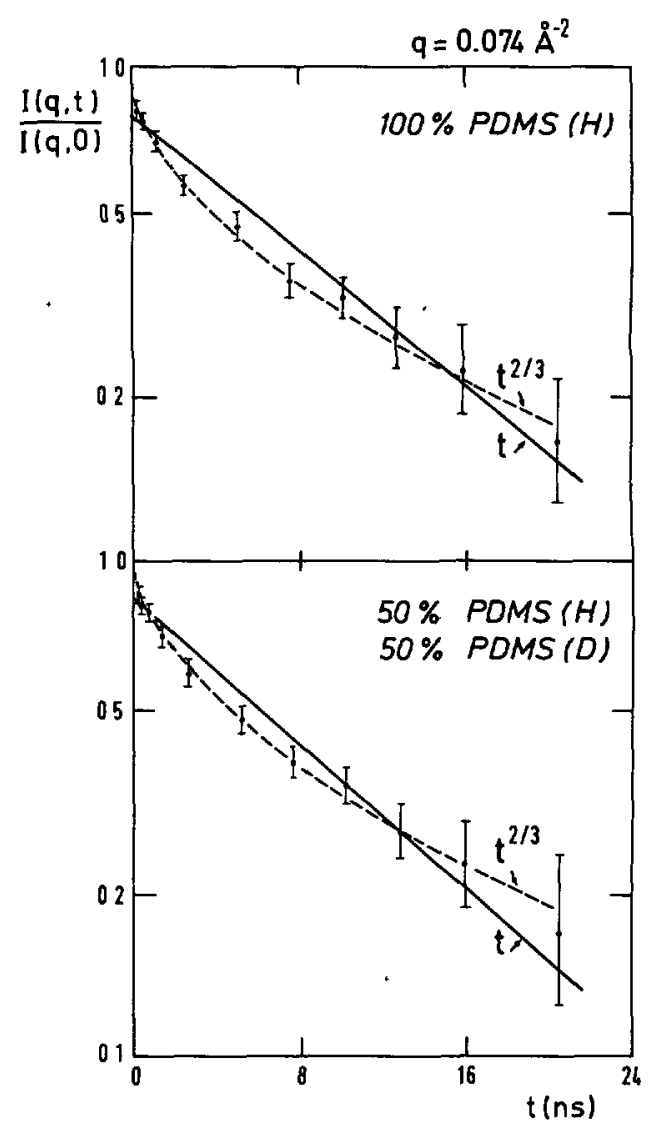

c)

Fig. 2 - Observed time decay of correlations in polymer concentration fluctuations a) Observations on sample (1) and (2) at a same value of $q\left(0042 \AA^{-1}\right)$. (๑) observed values of $\ln I(q, t) / I(q, 0)$ plotted against $t,(-)$ exponential decay, $(-)$ stretched exponential decay with exponent $1 / 2$. The model curves which are superposed to the data tend to show that the exponential decay better fits the data on concentration diffusion (sample (1)), and that the stretched exponential (exponent 1/2) better fits the data on inter-diffusion (sample (2)) b) Observations on sample (1) and (2) at a same value of $q$ $\left(0067 \AA^{-1}\right)$ Same remarks as in 1a) c) Observations on sample (1) and (2) at a same value of $q$ $\left(0074 \AA^{-1}\right)(\bullet)$ observed values of $\ln I(q, t) / I(q, 0)$ plotted against $t,(\longrightarrow)$ exponential decay, $(--)$ stretched exponential decay with exponent $2 / 3$ There is a change in behaviour with respect to $a$ ) and $b$ ) The model curves which are superposed to the data tend to show that the stretched exponential with power $2 / 3$ best fits the data associated both to sample (1) and (2) 


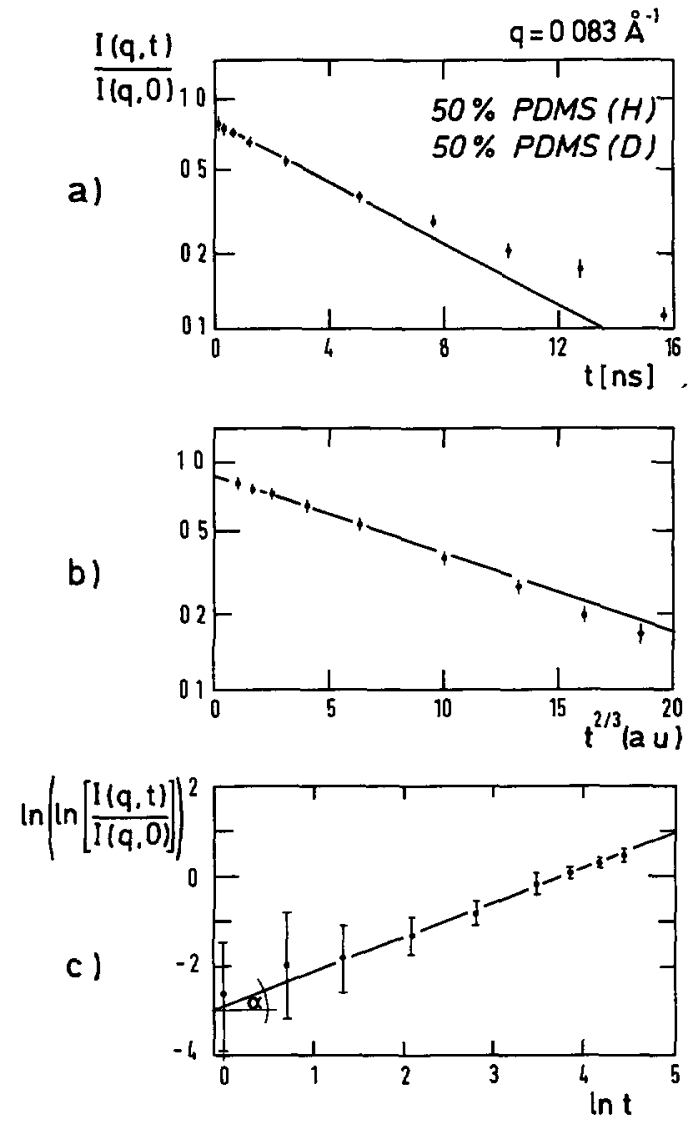

Fig 3. - Observed tıme decay of correlations in polymer concentration fluctuation Sample (2) $q=0.083 \AA^{-1}$ Here we try to determine the power law associated with the stretched exponential decay of the correlations a) Plot of $\ln I(q, t) / I(q, 0)$ against $t(\bullet)$ observed values , $(-)$ exponential decay b) Plot of $\ln I(q, t) / I(q, 0)$ against $t^{2 / 3}$. (๑) observed values; $(-)$ stretched exponential decay with power $2 / 3$ c) Double logarithmic plot. The slope $\alpha$ is found to be $0.73 \pm 005$.

$q \xi>1$. We therefore represent $\ln (I(q, t) / I(q, 0))$ as a function of $t^{2 / 3}$ and, for comparison, as a function of $t$ (Fig. 3).

We find that it is thus possible to discern the specific time correlation decay functions, which are associated with each type of polymer Brownian motion. In the next sections, we test in more detail the quantitative predictions of the models.

4.3 DiSPERSION CURVE OF THE RELAXATION TIMES - The decrease of the correlation function with time $t$ is charactenzed by one or several relaxation times. The relaxation times depend on the wave vector transfer $q$, and this dependence forms the dispersion relation. We wish to show, on a single figure, the dispersion relations obtained from experiments on the two samples. For this, we fit the data to the first cumulant approximation (Eq. (21)) which appears to be the only unified description, by taking the slope at the origin.

In figure 4 we plot ${ }^{\circ} \Gamma_{q} / q^{2}$ against $q$ The quantity ${ }^{\circ} \Gamma_{q} / q^{2}$ has the dimension of a Fickian diffusion coefficient. We note in figure 4 three characteristic domains for the dispersion relations. 
1) The domain, limited by $q \xi \leqslant 1$, of the results obtained with sample (1). Here - $\Gamma_{q} / q^{2}$ is a constant. It is associated with the concentration diffusion (Eq. (29)) and it is Identified with the cooperative diffusion coefficient, $D_{\text {coop}}$.

2) The domain, limited by $q \xi \geqslant 1$, for the results obtained with sample (1) and sample (2). We notice that the experiments carried separately on the two samples, coincide. In this range, - $\Gamma_{q} / q^{2}$ increases with $q$ following equation (27b).

3) The new complementary result is found in data obtained with sample (2) (the zero average contrast sample), for $q \xi \leqslant 1$, where equation (27a) is predicted. This is in agreement with the fact that in figure 4 the upper part of the dispersion curve, $q \xi<1$, splits into two branches as $q \xi \simeq 1$.

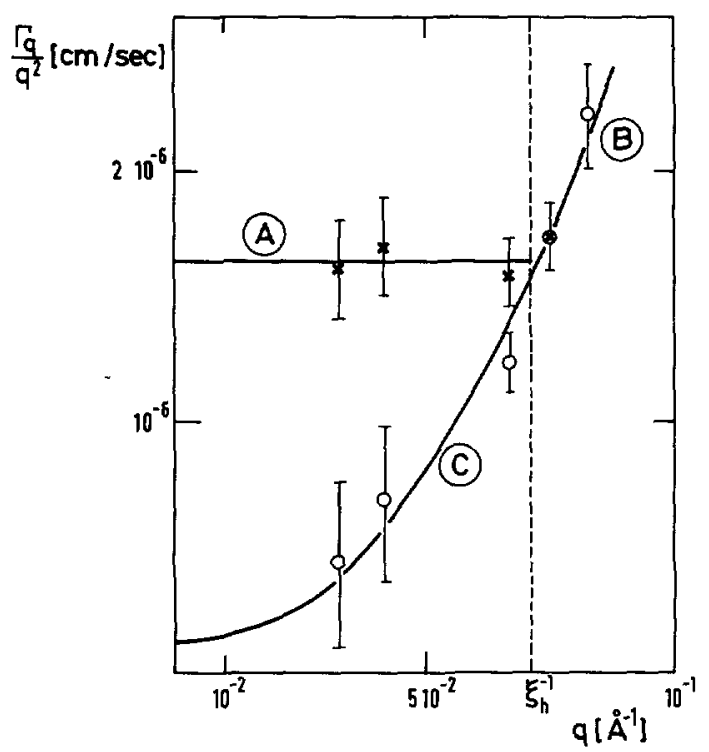

Fig 4 -Dispersion relation of the relaxation times observed on samples (1) and (2) Observed values of ${ }^{\circ} \Gamma_{q} / q^{2}$ are plotted against $q(\times)$ sample (1) PDMS(H), (O) sample (2) PDMS(D) + PDMS(H) $\underline{A}, \underline{B}$ : guide to the eye, $\underline{C}$ : dispersion curve estımated from formula (27a). Branches $A, B$ correspond to $I(q, 0)$ in figure 1 . Branches $\mathrm{C}, \mathrm{B}$ correspond to $I_{Z}(q, 0)$ in figure 1 .

This splitting is also consistent with the prediction [14]

$$
{ }^{\circ} \Gamma_{q} / q^{2}=\frac{D_{\text {self }}}{h(q, 0)}\left(1-\frac{\varphi \chi N}{2} h(q)\right)
$$

where $\varphi$ is the volume fraction of the chains $(\varphi=0.2)$. The parameter $\chi$ (Tab. I) accounis ior the specific H-D interaction. The quantity $\varphi \chi N / 2$ is here of the order of 0.1 (see Tab. I) and we shall neglect the effect of this interaction. As a result.

$$
{ }^{\circ} \Gamma_{q} / q^{2}=\frac{D_{\text {self }}}{h(q, 0)}\left(\simeq \frac{D_{\text {self }}}{2}\left(q R_{\mathrm{G}}\right)^{2}, q R_{\mathrm{G}}>1\right)
$$

We now study the vicinity of $\xi^{-1}$ in greater detall. 
4.4 Evaluation OF THE DIFFUSION COEFFICIENTS. - Here we abandon the first cumulant approximation for more precise definition of diffusion coefficients, each one corresponding to a specific case.

The relaxation associated whth cooperative diffusion is correctly given by the exponential decay function. Here the values ${ }^{\circ} \Gamma_{q} / q^{2}$ should be independent of $q$ and this is not incompatible with the data (Tab. II). Averaging the experimental values we find :

$$
D_{\text {coop }}={ }^{\circ} \Gamma_{q} / q^{2}=1.63 \times 10^{-6} \mathrm{~cm}^{2} \mathrm{~s}^{-1} \text {. }
$$

For the relaxation process related to the internal diffusive modes $(q \xi>1)$, we use the interpolation formula (27b) and we find by averaging values of table II :

$$
{ }^{\mathrm{z}} \Gamma_{q} / q^{3}=\mathfrak{D}=\frac{1}{6 \pi \eta_{\mathrm{s}} \beta} \simeq 1.52 \times 10^{-13} \mathrm{~cm}^{3} \mathrm{~s}^{-1}
$$

For the interdiffusion or self-diffusion in the range $q \xi \leqslant 1$, related with sample (2), we use the formula (27a) and (25).

Table II. - Experimental results

\begin{tabular}{|c|c|c|c|c|c|}
\hline$q$ & 33 & 42 & 67 & 7.5 & $83\left[10^{-2} \AA^{-1}\right]$ \\
\hline $\begin{array}{l}{ }^{\circ} \Gamma_{q} / q^{2} \\
{ }^{\mathrm{z}} \Gamma_{q} / q^{3}=\mathrm{D} \\
{ }^{\mathrm{R}} \Gamma_{q} / q^{4}=\mathrm{D}\end{array}$ & $\begin{array}{c}1.7 \pm 034 \\
\times 10^{-6} \mathrm{~cm}^{2} \mathrm{~s}^{-1}\end{array}$ & $\begin{array}{c}1.59 \pm 0.16 \\
\times 10^{-6} \mathrm{~cm}^{2} \mathrm{~s}^{-1} \\
\\
3.052 \pm 07 \\
\times 10^{-20} \mathrm{~cm}^{4} \mathrm{~s}^{-1}\end{array}$ & $\left|\begin{array}{c}16 \pm 02 \\
\times 10^{-6} \mathrm{~cm}^{2} \mathrm{~s}^{-1} \\
\\
\\
213+0.28 \\
\times 10^{-20} \mathrm{~cm}^{4} \mathrm{~s}^{-1}\end{array}\right|$ & $\begin{array}{c}147 \pm 023 \\
\times 10^{-13} \mathrm{~cm}^{3} \mathrm{~s}^{-1}\end{array}$ & $\begin{array}{c}167 \pm 0.21 \\
\times 10^{-13} \mathrm{~cm}^{3} \mathrm{~s}^{-1}\end{array}$ \\
\hline
\end{tabular}

Inverse relaxation ttmes

Cooperative diffusion: $D_{\text {coop }}=\frac{1}{6 \pi \eta_{\mathrm{s}} \beta \xi}=163 \times 10^{-6} \mathrm{~cm}^{2} \mathrm{~s}^{-1}$

Self and inter-diffusion : $D_{\text {self }}(\rho \rightarrow 0)=2.1 \times 10^{-7} \mathrm{~cm}^{2} \mathrm{~s}^{-1}[40]$

$$
\begin{aligned}
& D_{\text {self }}(\rho \rightarrow 0)=5.2 \times 10^{-7} \mathrm{~cm}^{2} \mathrm{~s}^{-1}[41] \\
& D_{\text {self }}\left(\rho=02 \mathrm{~g} \mathrm{~cm}^{-3}\right)=5.7 \times 10^{-8} \mathrm{~cm}^{2} \mathrm{~s}^{-1}[29]
\end{aligned}
$$

From the relation ${ }^{\mathrm{R}} \Gamma_{q} / q^{4}=\mathfrak{D}=R_{\mathrm{G}}^{2}{ }^{\mathrm{R}} D_{\text {self }}$ and table II, we expect the value of ${ }^{\mathrm{R}} D_{\text {self }}$, the center of mass diffusion constant in the « Rouse » regime :

$$
{ }^{\mathrm{R}} D_{\text {self }}\left(\rho=02 \mathrm{~g} / \mathrm{cm}^{3}\right)=57 \times 10^{-8} \mathrm{~cm}^{2} \mathrm{~s}^{-1}
$$

\section{Discussion.}

Our main results are shown in figures $2,3,4$ which account for the dynamics of the polymer solution in the vicinity of $\xi^{-1}$. The new complementary information is given by branch $\mathrm{C}$ in the dispersion relation and the corresponding time dependences of the correlation function (Fig. 2a, b). We now examine the consistency of these results with theoretical prediction. 
5.1 TIME INTERVAL ASSOCIATED WITH STRETCHED EXPONENTIAL BEHAVIOUR. - In the theory [19] of polymer dynamics in dilute solutions, the decay of the correlation function appears as a stretched exponential, in an interval

$$
\left(\Gamma_{q}\right)^{-1} \leqslant t \leqslant R^{2} / D_{\text {self }}
$$

In the « Rouse " regime, 1.e. for sample (2) and $q=6.7 \times 10^{-2} \AA$, this condition becomes (see tab. II)

$$
{ }^{\mathrm{R}} \Gamma_{q}^{-1}\left(\simeq 10^{-8} \mathrm{~s}\right) \leqslant t<10^{-3} \mathrm{~s} .
$$

In the « $Z_{1 \mathrm{~mm}}$ regime », 1.e for samples (1) and (2), and $q=7.5 \times 10^{-2} \AA^{-1}$, this condition becomes

$$
{ }^{\mathrm{z}} \Gamma_{q}^{-1}\left(\simeq 10^{-8} \mathrm{~s}\right) \leqslant t<10^{-5} \mathrm{~s}
$$

The fact that stretched exponentials are observed in the time intervals of figures 1 and 2 , is therefore not in contradiction with theory. However we notice that the fits are good on larger ranges than predicted by (37) and (38).

5.2 The dispersion relation in figure 4 displays a cross-over between charactenstic behaviours in sem1-dilute and dilute regimes (branches A and B in Fig. 4). The cross-over takes place at an inverse distance, $1 / \xi_{H}$, which is smaller than the result $1 / \xi$ defined from the static structure function. Also, the cross-over is much sharper than predicted by the calculations based on the mode decoupling theory [19].

This astonishing fact, already observed in several experiments, has not received any explanation.

5.3 The last part of the discussion concerns the values of the diffusion coefficients derived from experımental data.

If we admit that the viscosity of the solvent does not effectively change as concentration increases, then the correlation length $\xi$ can be derived from the cooperative diffusion $D_{\text {coop }}$ (Eq. (28))

$$
D_{\text {coop }}=\frac{1}{6 \pi \eta_{\mathrm{s}} \beta \xi_{\mathrm{H}}}=163 \times 10^{-6} \mathrm{~cm}^{2} \mathrm{~s}^{-1}
$$

and this gives $\xi_{H}=22 \AA$. This result is different from observations of the static correlations length, which is $\xi=10 \AA$. Introducing this value into (39) gives

$$
D_{\text {coop }}=3.6 \times 10^{-6} \mathrm{~cm}^{2} \mathrm{~s}^{-1} \text {. }
$$

Another discrepancy is found in the values of the inverse of the internal mode diffusion. We have derived from the data

$$
\mathscr{D}={ }^{\mathrm{z}} \Gamma_{q} / q^{3}=1.5 \times 10^{-13} \mathrm{~cm}^{3} \mathrm{~s}^{-1}
$$

whereas the calculation gives

$$
D={ }^{\mathrm{Z}} \Gamma_{q} / q^{3}=\frac{1}{6 \pi \eta_{\mathrm{s}} \beta}=3.6 \times 10^{-13} \mathrm{~cm}^{3} \mathrm{~s}^{-1} .
$$

We note that this discrepancy and the preceding one are similar The introduction of the hydrodynamic length $\xi_{H}$ and $R_{H}$ should thus correspond to the introduction of an effective viscosity $\eta_{\mathrm{S}}^{\mathrm{e}}$ which replaces $\eta_{\mathrm{s}}$ in equation (41) 
Finally we discuss values of the self-diffusion coefficient extrapolated from data. In the range $q \xi \leqslant 1$, and in semi-dilute solution, the dynamical structure function of the single chain has a characteristic behaviour, different from the one observed in isolated chains. Our data are consistent with the prediction that the hydrodynamic interaction is screened [15, 19], and that the chain moves in an effective medium [19] . it obeys a "Rouse » equation in the time interval (37). (This is at variance [21] with earlier results [12].)

Now, for tımes longer than considered in (37), entanglements are predicted to characterize the diffusive motion. This effect can be evaluated, by comparing the self-diffusion in the effective medium to the self-diffusion of the chain in pure solvent. For this we determine $D_{\text {self }}(\rho \rightarrow 0) \sim \frac{{ }^{\mathrm{Z}} \Gamma_{q}}{q^{3}} \cdot \frac{1}{R_{\mathrm{G}}}$. Using for ${ }^{\mathrm{z}} \Gamma_{q} / q^{3}$ either equation (40) or (41), we obtain the two values, given in table II, from the data of branch B in the dispersion curve. Using also the value of $D_{\text {self }}\left(\rho=0.2 \mathrm{~g} / \mathrm{cm}^{3}\right)$ the data of branch C, we expect (Eq (24)) the ratio.

$$
\frac{D_{\text {self }}(\rho \rightarrow 0)}{D_{\text {self }}\left(\rho=0.2 \mathrm{~g} / \mathrm{cm}^{3}\right)}=\left(\frac{C}{C^{*}}\right)^{7 / 4} \approx 6.6 \text {. }
$$

This number is not far from the two experimental values obtained from table II.

In conclusion, the observations made on the two sem1-dilute solutions at same polymer concentration but at different isotopic fractions, show distinct transport behaviours on each side of the inverse of the "dynamic " mesh size $q=\xi_{\mathrm{H}}^{-1}$.

Inter-diffusion exists as such not only in the natural range $q R_{\mathrm{G}} \simeq 1$, but also in the entire interval $q \xi \leqslant 1$. In this experiment inter-diffusion coincides with the self-diffusion. The situation will be different in a bidisperse system, made of longer and shorter chains

Finally, it is of interest to compare the effects of repulsive and attractive interactions on the dispersion curve of the inter-diffusive mode. In the repulsive case, which we have discussed here, the inter-diffusive branch is below the cooperative branch. On the contrary, in the attractive case of copolymer, which are attached together and represent a kind of attraction (Ref [11]), the inter-diffusive branch is above the "cooperative" branch.

\section{Acknowledgments.}

We thank M. Lambert, B. Farago and F. Mezei for their assistance in the experiment. We are grateful to $H$. Benoit and $M$. Duval for enlightening discussions in the first stage of the project.

Note added in proof. - A companson with former data can first be made directly of the lower plots of figures $2 a$ and $2 b$ with figure 9 of reference [12], corresponding also to measurements of $H^{1}(q, t)$ with a solvent of similar viscosity. For concentrations ca $20 \%$, the decays are similar Parameter $\mathfrak{D}={ }^{\mathrm{R}} \Gamma_{q} / q^{4}$ (Eq. (27a)) can be compared within a factor 36 , to parameter of $W \sigma^{4}$ (Eq. (2) of Ref. [12]). $W$ is an elementary frequency and $\sigma$ an elementary time, but conceptually the relevant quantity in the Rouse model is the product $W \sigma^{4}$. It is found equal to $3 \times 10^{13} \AA^{4} \mathrm{~s}^{-1}=30 \times 10^{-20} \mathrm{~cm}^{4} \mathrm{~s}^{-1}$ in pure melt, $100 \times 10^{-20} \mathrm{~cm}^{4} \mathrm{~s}^{-1}$ for $50 \%$ polymer, and $650 \times 10^{-20} \mathrm{~cm}^{4} \mathrm{~s}^{-1}$ for $20 \%$ using the $c^{-2}$ law found by the authors between $100 \%$ and $50 \%$ Dividing by 36 gives a value 6 times larger to our $\mathcal{D}=3 \times 10^{-20} \mathrm{~cm}^{4} \mathrm{~s}^{-1}$ Values in the Zimm regime [12] of $\omega_{\mathrm{c}} / q^{3}=150 \mu \mathrm{eV} \AA \AA^{3}=$ $23 \times 10^{-13} \mathrm{~cm}^{3} \mathrm{~s}^{-1}$ corresponds to $1.5 \times 10^{-13} \mathrm{~cm}^{3} \mathrm{~s}^{-1}$ given here for $\mathfrak{D}={ }^{\mathrm{Z}} \Gamma_{q} / q^{3}$ $\left(\omega_{\mathrm{c}} \sim{ }^{\circ} \Gamma_{q}={ }^{\mathrm{Z}} \Gamma_{q}\right)$. Values of $D_{\text {coop }}$ can be found in reference [23]:4 $\times 10^{-6} \mathrm{~cm}^{2} \mathrm{~s}^{-1}$ for $20 \%$, to be compared with our value $2 \times 10^{-6} \mathrm{~cm}^{2} \mathrm{~s}^{-1}$, slightly lower. The crossover value between the regime $A$ and $B, \xi^{-1}$, is the same one. 


\section{References}

[1] Edwards S F, Proc Phys Soc London 85 (1965) 613

[2] DES CloizeAuX $\mathbf{J}$ and JANNINK $\mathbf{G}$, Les polymères en solution leur modélisation et leur structure (Editions de Physique, Les Ulis) 1987

[3] Polymer motion in dense systems D. Richter and T Sprınger Ėds (Sprınger Proc Phys vol 29 (Berlin)) 1988

[4] Adam M, Delsanti M and Jannink G, $J$ Phys France Lett 37 (1976) L-53

[5] Ewen B, Richter D., Hayter J B. and Lehner B., J Polym. Sct., Polymer Lett Ed 20 (1981) 233

[6] Leger L., Hervet H and Rondelez F, Macromolecules 14 (1981) 1732.

[7] Richter D., Hayter J, Mezei F. and Ewen B, Phys Rev Lett 41 (1978) 1484

[8] Benmouna M, Benoit H, Duval M and Akcasu Z., Macromolecules 20 (1987) 1107

[9] Broseta D, Thesis Paris 1988

[10] Borsali R, Duval M and Benmouna M., Macromolecules 22 (1989) 816.

[11] Borsali R, Benoit H., Legrand J F., Duval M , Picot C, Benmouna M and farago B, Macromolecules 22 (1989) 4119.

[12] Richter D., Binder K, Ewen B. and Stuhn, J Phys Chem. 88 (1984) 6618

[13] MEZEI F., $Z$ Phys 255 (1972) 146

[14] BORSALI R, Thesis (Strasbourg) 1988

[15] Jannink G and De Gennes P G, $J$ Chem Phys 48 (1968) 2260

[16] de Gennes P G, Scaling Concepts in Polymer Physics (Cornell University Press, Ithaca, NY) 1979

[17] DE GENNES P. G, Physics 3 (1967) 37.

[18] Dubois-Violette E and De Gennes P G, Physics 4 (1967) 181

[19] Dol M and Edwards S F, The Theory of Polymer Dynamics (Clarendon Press, Oxford) 1986

[20] Friedman H L and Mills R, J Solution Chem. 10 (1981) 395

[21] Richter D, Private Communication

[22] LAPP A, Picot C and BenoIt H., Macromolecules 18 (1985) 2437.

[23] Ewen B., Richter D, Hayter B., Lehner B, J Pol Sct, Pol Lett Ed 20 (1982) 233-240 